\title{
Comportamiento productivo del Pennisetum sp a la aplicación de 10-30-10, gallinaza y urea en el cantón Morona Santiago
}

\author{
Productive behavior of Pennisetum sp to the application of 10-30-10, \\ gallinaza and urea in the canton Morona Santiago
}

\author{
Víctor Huebla Concha. ${ }^{1}$, Luis Condo Plaza. ${ }^{2}$, Luis Arias Alemán. ${ }^{3} \&$ Nancy Tapia \\ Cabrera. ${ }^{4}$
}

\begin{abstract}
.
\end{abstract}
DOI: https://doi.org/10.33262/concienciadigital.v4i1.2.1592

Introduction. Pennisetum sp is a tropical grass that is characterized by its high productive yield being demanding to fertilization, reason for which the objective was set. To determine the productive behavior of Pennisetum sp to the application of 10-30-10, poultry manure and urea in the Morona canton. Methodology, the present work was developed at an altitude of 1016 meters above sea level, and an ambient temperature between 15 to $32{ }^{\circ} \mathrm{C}$, whose meteorological conditions were $80 \%$ relative humidity, 3000 to $4000 \mathrm{~mm}$ of rainfall and 12 hours/day of heliophany; $192 \mathrm{~m}^{2}$ of surface were used in which the Pennisetum sp (maralfalfa) crop was established and on which the treatments were applied: $1000 \mathrm{~kg} / \mathrm{ha}$ of 10-30-10 (T1), $10 \mathrm{Tm} /$ ha of chicken manure (T2) and 10 Tm of chicken manure plus $1000 \mathrm{~kg}$ of 10-30-10/ ha (T3); in each treatment $200 \mathrm{~kg}$ of Urea were applied, against a control, with four replications, which were distributed considering the mathematical model corresponding to the Completely Randomized Block Design, the crop information was: plant height at 30, 60 and 90 days, green forage

\footnotetext{
${ }^{1}$ Investigadores del proyecto GIDES-ESPOCH, Escuela Superior Politécnica de Chimborazo-ESPOCH, carrera: Zootecnia, provincia Morona Santiago, correo: zvictorh@yahoo.es; https://orcid.org/0000-0002$3597-5890$

2 Universidad Regional Amazónica IKIAM, carrera: Biocomercio, provincia: Napo, Centro Latinoamericano de Estudios de Problemáticas Lecheras (CLEPL) - Argentina Universidad el Rosario, Investigador IIINOCA - México correo: lac_plaza@yahoo.com. https://orcid.org/0000-0001-9625-9620.

${ }^{3}$ Investigadores del proyecto GIDES-ESPOCH, Escuela Superior Politécnica de Chimborazo-ESPOCH, carrera: Zootecnia, provincia Morona Santiago, correo: zvictorh@yahoo.es; https://orcid.org/0000-00023597-5890 luis.arias@espoch.edu.ec. https://orcid.org/0000-0001-8262-8157

${ }^{4}$ Investigadores del proyecto GIDES-ESPOCH, Estudiante Investigador de la Escuela Superior Politécnica de Chimborazo-ESPOCH, carrera: Zootecnia, provincia: Morona Santiago, nancyeu93@outlook.com. https://orcid.org/0000-0003-3755-9505
} 
production and dry matter. Results. It was demonstrated that the application of $10 \mathrm{Tm}$ of poultry manure, $1000 \mathrm{~kg}$ of $10-30-10$ plus $200 \mathrm{~kg}$ of urea allowed to register heights at 30, 60 and 90 days of $0.66,1.24$ and 1.26 meters and a production of green forage and dry matter of 65.20 and $13.04 \mathrm{Tn} / \mathrm{ha}$, superior to the rest of the fertilization system; it is concluded that the use of fertilizer 10-30-10 + urea in doses of 1000 and $2000 \mathrm{~kg} / \mathrm{ha} / \mathrm{crop}$ allows a higher forage production.

Key words: Evaluation, growth, maralfalfa, fertilizer.

\section{Resumen.}

Introducción. El Pennisetum $s p$ es una gramínea tropical que se caracteriza por su alto rendimiento productivo siendo exigente a la fertilización, razón por la que se plateó el objetivo. Determinar el comportamiento productivo del Pennisetum $s p$ a la aplicación de 10-30-10, gallinaza y urea en el cantón Morona. Metodología, el presente trabajo se desarrolló a una altura de 1016 metros sobre el nivel del mar, y una temperatura ambiental entre 15 a $32{ }^{\circ} \mathrm{C}$, cuyas condiciones meteorológicas fueron $80 \%$ de humedad relativa, 3000 a $4000 \mathrm{~mm}$ de pluviometría y 12 horas/luz de heliofanía; se utilizó $192 \mathrm{~m}^{2}$ de superficie en el cual se estableció el cultivo de Pennisetum sp (maralfalfa) sobre el cual se aplicó los tratamientos: $1000 \mathrm{~kg} / \mathrm{ha}$ de 10-30-10 (T1), $10 \mathrm{Tm} /$ ha de gallinaza (T2) y 10 Tm de gallinaza mas $1000 \mathrm{~kg}$ de 10-30-10 / ha (T3); en cada tratamiento se aplicó 200 $\mathrm{kg}$ de Urea, frente a un control, con cuatro repeticiones, los cuáles fueron distribuidos considerando el modelo matemático que corresponde al Diseño de Bloques Completamente al Azar, la información del cultivo fue: altura de planta a los 30, 60 y 90 días, producción de forraje verde y materia seca. Resultados. Demostrándose que la aplicación de 10 Tm de gallinaza, $1000 \mathrm{~kg}$ de10-30-10 más $200 \mathrm{~kg}$ de urea permitió registrar alturas a los 30, 60 y 90 días de $0.66,1.24$ y 1,26 metros y una producción de forraje verde y materia seca de 65.20 y $13.04 \mathrm{Tn} / \mathrm{ha}$, superior al resto de Sistema de fertilizaciones; se concluye que la utilización de fertilizante 10-30-10 + urea en dosis de 1000 y $2000 \mathrm{~kg} / \mathrm{ha} /$ corte permite una mayor producción de forraje.

Palabras clave: Evaluación, crecimiento, maralfalfa, fertilizante

\section{Introducción}

En la provincia de Morona Santiago ubicada al sur de la Amazonía Ecuatoriana cuenta con doce cantones cuya principal actividad económica es la ganadería de carne, para lo cual requiere de pastizales como materia prima para el propósito manifestado.

Si bien es cierto, el Pennisetum sp (Maralfalfa), es la principal fuente de alimento del Ganado en el cantón Morona, mientras que el maralfalfa constituye una alternativa para la producción de leche y carne, sus características volumétricas y nutritivas de producción 
permiten impulsar este cultivo, aunque, esta eficiencia exige mayor cantidad de fertilización por corte y por año (Correa et al, 2014).

La mayoría de los pastos incluidos el Pennisetum sp (maralfalfa), se caracterizan por tener una alta producción de forraje verde y materia seca, por su adaptación y el valor nutritivo (Cabascango, 2018). Sin embargo, es necesario tomar en cuenta la eficiencia en la producción ganadera, estableciendo cultivares adaptados con altos rendimientos por unidad de superficie que contribuyan a mantener una buena capacidad de carga y consecuentemente incrementar la producción de carne y/o leche por unidad de superficie. Estas características son las que distinguen al género Pennisetum sp (Duran, F. 2016).

El Pennisetum sp (Maralfalfa), es un pasto perenne con alta productividad, las raíces son fibrosas y adventicias se forman de los nudos inferiores de los tallos expuestos superficialmente, cerca de la base el espacio entrenudo es más corta en relación con los entrenudos de la parte superior, una de las características es que no poseen vellosidades. Se desarrolla bien, altitudes inferiores a los 2600 metros sobre el nivel del mar y precipitaciones entre los 1000 y $4000 \mathrm{~mm} / \mathrm{anuales}$, se adapta a suelos de $\mathrm{pH}$ entre ligeramente ácidos y neutros $(5,5$ y 7,4$)$ y de fertilidad media a alta, pero, no tolera la saturación de aluminio, ni el encharcamiento, produce hasta 60 toneladas de biomasa seca por hectárea por corte, con un contenido de proteína cruda de 8 a $16 \%$ y una digestibilidad del 55 al 70\% (STDF, 2013, citado por Cerdas, R. 2015).

El crecimiento de los pastos involucra cuatro procesos primarios: la aparición de hojas, la aparición de tallos, la formación de tallos verdaderos y la aparición de raíces. El ambiente caracterizado por el suelo y el clima, tienen gran influencia en el crecimiento y desarrollo de las plantas y por lo tanto en su rendimiento; sin embargo, la disponibilidad de los recursos ambientales está supeditada a factores como tipo de suelo, altitud, vientos y decisiones de manejo agronómico. La luz solar y la temperatura afectan la tasa de crecimiento de las especies forrajeras, así como la tasa y tiempo de desarrollo de los estados fenológicos (Calzada, J. 2007).

Al considerar que el Pennisetum sp es un pasto exigente a la fertilización principalmente orgánica en el presente trabajo se utilizó gallinaza, 10-30-10 y ures, los cuales aportan una gran cantidad de maccroelementos minerales que favorecen el desarrollo del pasto.

Así se puede manifestar que la gallinaza es un subproducto de la industria avícola, debido a su producción intensiva tiene el potencial de proveer además de huevo y carne; los materiales de desecho orgánico y de calidad toma el nombre de gallinaza el mismo tiene grandes ventajas para incrementar la producción de los cultivos agrícolas o pastizales para la producción pecuaria, entre los elementos más importantes en que dispone este desecho avícola es el N, P y K, e incremento de la materia orgánica del suelo (Estrada, 2005). 
La gallinaza es un abono de excelente calidad, su eficiencia se logra utilizando las excretas descompuestas, previo a un tratamiento de ventilación para obtener un abono con alta disponibilidad de nutrientes orgánicos; El aireado y secado de las heces deben realizarse en zonas donde se protegen de las lluvias. La forma piramidal es la más utilizada la misma, permite el escurrimiento de la humedad en menor tiempo (Estrada, 2005). Finalmente, la gallinaza posee nutrientes que generan beneficios para los cultivos, siendo necesario realizar el análisis de y conocer los compuestos para su correcta aplicaron (Estrada, 2005).

La Urea más químicamente conocida como Carbamina es un fertilizante de origen orgánico. Entre los fertilizantes sólidos la urea de fuente Nitrogenada y de más alta concentración que posee grandes ventajas en términos económicos y de manejo de cultivos además de fuente proteica en rumiantes, este producto es altamente demandantes de Nitrógeno. También se conoce como Carbonildiamida, Ácido Carbamídico ó Amida Alifática (PACIFEX, 2018).

En el suelo se hidroliza con rapidez por efecto de la enzima "ureasa" produciéndose iones de Amonio y de Amoniaco. Al aplicar en superficialmente en los suelos se pierde Amoniaco (NH3) por volatilización, el Amonio (NH+ 4) además es absorbido por las arcillas y la materia orgánica del suelo y eventualmente nitrificado ó absorbido directamente por las plantas (PACIFEX, 2018).

En las plantas se absorbe la mayoría cantidad del Nitrógeno en forma de iones Amonio $(\mathrm{NH}+4)$ o Nitrato (NO3) y en muy pequeña proporción se obtiene aminoácidos solubles en agua. Los cultivos en la mayor parte absorben el Nitrógeno como nitratos, sin embargo, estudios demuestran que los cultivos usan cantidades importantes de Amonio del suelo. En el proceso de Nitrificación del $(\mathrm{NH}+4)$ en $(\mathrm{NO} 3)$, se liberan iones $\mathrm{H}+$, produciéndose acidez del suelo (PACIFEX, 2018).

La Urea como tal, tiene una diversidad de usos y aplicaciones. Siendo indispensable en la producción de fórmulas balanceadas de fertilización, además se puede aplicar directamente al suelo por su alta solubilidad se puede aplicar en agua como aporte de nitrógeno en formulas NPK foliares considerado como fertirriego. En aplicaciones foliares es muy importante utilizar Urea libre de Biuret (PACIFEX, 2018).

Uno de los fertilizantes químicos completos es el 10-30-10 cuya presentación es granular con una alta disponibilidad de fósforo y contenidos complementarios de nitrógeno y potasio. Su uso es común en diversos cultivos anuales o de ciclo corto, en fertilizaciones de establecimiento, así como de mantenimiento en las especies perennes o cíclicas (Yara, 2018).

El 10-30-10 actúa efectivamente desde las raíces en las diferentes fases fenológicas hasta obtener los frutos y semillas; el nitrógeno de este abono: estimula el crecimiento, permite 
un color verde intenso a las hojas y mejora su calidad; aumenta el contenido de proteínas en la estructura vegetal, permite una mayor producción de frutos y semillas y se considera un nutriente de los microbios del suelo. El fósforo estimula el desarrollo de la raíz y el crecimiento de la planta; desarrolla aceleradamente la planta y permite que sea el vegetal vigoroso en las plantas jóvenes, estimula la formación de flores y maduración de los frutos, es indispensable en la formación de la semilla. Y el potasio propicia el vigor y resistencia a la presencia de enfermedades, su presencia evita la caída de las plantas en interacción con el $\mathrm{Ca}$ y el $\mathrm{Mg}$; permite soportar las condiciones adversas del clima y permite la formación, transporte y de azucares y almidones (FERTIZA, 2020).

Por lo señalado anteriormente, se planteó el objetivo: analizar el crecimiento del pasto "Maralfalfa" (Pennisetum sp.) desde el establecimiento hasta el momento de la cosecha utilizando diferentes fertilizaciones.

\section{Materiales y métodos}

El comportamiento productivo del Pennisetum sp a la aplicación de 10-30-10, gallinaza y urea en el cantón Morona Santiago de la provincia Morona Santiago - Ecuador, se realizó a una altura de $1100 \mathrm{msnm}$. una temperatura de $18-23{ }^{\circ} \mathrm{C}$, humedad relativa entre 897 - $90 \%$, una precipitación de 1900 a $2300 \mathrm{~mm}$, una evaporación de $255 \mathrm{~mm} / \mathrm{año}$; para lo cual se utilizó el método científico, analítico sintético, se adecuó un espacio de $192 \mathrm{~m}^{2}$, y se procedió con el establecimiento del cultivo del maralfalfa, iniciando con la preparación del suelo, la fertilización y posteriormente con el manejo del cultivo, evitando se propague las malezas a través de método mecánico de desbroce para evitar la contaminación del ambiente al usar químicos, cuya práctica evita la competencia de absorción de nutrientes con el pasto propósito de la investigación.

En el terreno en el cual se estableció la investigación se estructuró cuatro bloques y dentro de ellos se distribuyó los cuatro tratamientos al azar, en donde se colocaron las diferentes unidades experimentales al azar y aplicar el modelo matemático que corresponde al Diseño de Bloques Completamente al Azar con tres tratamientos frente al testigo y cuatro repeticiones que se ajusta al siguiente modelo matemático: $Y i j=u+T i+R j+E i j:$ donde Yij: es la variable respuesta, u: es la media general, Ti: es el efecto de los i-ésimos tratamientos, Rj; se refiere a los j-ésimos bloques y Eij, el efecto de la aleatorización de los tratamientos dentro de cada uno de los bloques.

La aplicación del fertilizante de establecimiento se realizó de la siguiente manera: el tratamiento (T1) $1000 \mathrm{~kg}$ de 10-30-10 + $200 \mathrm{~kg}$ de urea por hectárea; el T2: $10 \mathrm{Tm} / \mathrm{ha}$ de gallinaza, + urea 200kg/ha, y el T3, $10 \mathrm{Tm}$ de gallinaza $+1000 \mathrm{Kg} / \mathrm{ha} 10-30-10+$ urea $200 \mathrm{~kg} / \mathrm{ha}$ y posteriormente se fue observando y midiendo el pasto conforme se estableció en las mediciones experimentales. Los resultados obtenidos fueron analizados mediante el análisis de la varianza (ADEVA) y la prueba estadística de separación de medias según la teoría de Tukey $(\mathrm{p}<0.05)$. 
Las mediciones experimentales tomadas durante la investigación fueron: altura de la planta a los 30, 60 y 90 días posterior al corte $(\mathrm{cm})$, la producción de forraje verde y materia seca de la vegetación en $\mathrm{kg} / \mathrm{m}^{2}$ y $\mathrm{Tm} / \mathrm{ha}$, y el momento óptimo de aprovechamiento del pasto.

\section{Resultados y discusión}

La altura del Pennisetum spp (maralfalfa) a los 30 días, al aplicar los tratamientos (T1) $1000 \mathrm{~kg}$ de 10-30-10 + $200 \mathrm{~kg}$ de urea por hectárea; (T2) 10 Tm/ha de gallinaza más urea $200 \mathrm{~kg} / \mathrm{ha}$, y (T3) $10 \mathrm{Tm}$ de gallinaza más $1000 \mathrm{Kg} / \mathrm{ha} 10-30-10$ más urea $200 \mathrm{~kg} / \mathrm{ha}$ fue de $0.64,0.62$ y $0.66 \mathrm{~m}$ respectivamente, diferenciándose significativamente $(\mathrm{p}<0.01)$ del tratamiento control con el cuál se alcanzó $0.54 \mathrm{~m}$. Transcurrido los 60 días, la aplicación de $1000 \mathrm{~kg}$ de 10-30-10 + $200 \mathrm{~kg}$ de urea por hectárea permitió obtener 1.28 $\mathrm{m}$ de altura; la utilización de $10 \mathrm{Tm} /$ ha de gallinaza más urea $200 \mathrm{~kg} /$ ha registró $1.24 \mathrm{~m}$ de altura, y al fertilizar con 10 Tm de gallinaza más $1000 \mathrm{Kg} /$ ha 10-30-10 más urea 200 $\mathrm{kg} / \mathrm{ha}$ se determinó $1.31 \mathrm{~m}$ de altura, los cuales difieren significativamente $(\mathrm{p}<0.01)$ tratamiento testigo con el cual se alcanzó $1.07 \mathrm{~m}$. Finalmente la altura del pasto maralfalfa a los 90 días se determinó que al utilizar $1000 \mathrm{~kg}$ de 10-30-10 más $200 \mathrm{~kg}$ de urea por hectárea; $10 \mathrm{Tm} / \mathrm{ha}$ de gallinaza, más urea $200 \mathrm{~kg} / \mathrm{ha}$, y $10 \mathrm{Tm}$ de gallinaza $+1000 \mathrm{Kg} / \mathrm{ha}$ 10-30-10 más urea $200 \mathrm{~kg} / \mathrm{ha}$, registró 2.03, 1.96 y $2.07 \mathrm{~m}$ respectivamente, diferenciándose significativamente $(\mathrm{p}<0.01)$ del tratamiento control, con el cuál se alcanzó $1.71 \mathrm{~m}$; de esta manera se observa que, la aplicación de fertilizantes en diferentes dosis es superior estadísticamente al tratamiento control, por lo que es necesario utilizar fertilizaciones para alcanzar mayor altura y consecuentemente producción de forraje verde y materia seca. Calzada, J. (2007) reporta que al analizar el crecimiento del maralfalfa aplicando una dosis de fertilizante 120-80-00 kg/ha de $\mathrm{N}$ y P2O5, respectivamente en dos aplicaciones registró una altura máxima de 2,3 metros del pasto maralfalfa a los 151 días luego del establecimiento. Hinojosa, L. (2010) al determinar la producción del maralfalfa registró una altura de 120,01 cm, valor similar al registrado en el presente estudio al evaluar a los 60 días. Así mismo se señala que la fertilización y la disponibilidad de agua en forma de lluvia durante el establecimiento y mantenimiento del cultivo entre los 90 y 110 días, incrementan la producción de forraje verde y el tamaño de las hojas y tallos sin afectar su composición química (Correa et al., 2015). Se puede atribuir a que los fertilizantes tales como el 10-3010 a más de disponer de nitrógeno, fósforo y potasio, tiene magnesio, boro, zing; razón por lo que se denomina un abono completo, el mismo que se debe utilizar en base a la disponibilidad de nutrientes del suelo y los requerimientos del cultivo (Fertiza, 2020).

La aplicación de urea permite la disponibilidad de nitrógeno a la planta, elemento fundamental en el desarrollo, principalmente de las gramíneas, las cuales requieren de nitrógeno que ayuda al crecimiento de la planta, por lo tanto, ayuda en la altura de la maralfalfa puesto que el $\mathrm{N}$ influye en la síntesis de la clorofila y forma parte de esta 
molécula. Cantidades adecuadas de $\mathrm{N}$ en la maralfalfa, permite la producción de hojas de color verde oscuro debido a tienen alta concentración de clorofila. El pigmento de clorofila absorbe la energía de la luz necesaria para iniciar la fotosíntesis (Rincón y Ligarreto, 2010). Conociendo que estos suelos son bajos en nitrógeno lo que compensa la disponibilidad tanto de urea como la gallinaza para satisfacer los requerimientos de la planta, aunque los abonos inorgánicos apenas la tercera parte muestran su eficiencia en los cultivos (Corrales, et al. 2016).

Es necesario recordar que no solo la urea es necesario en el cultivo de maralfalfa para satisfacer los requerimientos nutricionales, sino que, es imprescindible aplicar otros fertilizantes orgánicos como la gallinaza, la misma que permite incluir $\mathrm{Ca}, \mathrm{P}, \mathrm{Mg}$, y $\mathrm{K}$ de siendo necesario cantidades de $0,37 \%, 0,50 \%, 0,37 \%$ y $6,28 \%$ respectivamente (Correa, 2006), cuando se cosecha este pasto a los 56 días. De la misma manera citan que los requerimientos de $\mathrm{N}, \mathrm{P}, \mathrm{K}, \mathrm{Ca}, \mathrm{y} \mathrm{Mg}$ en el cultivo de gramíneas es de 1,0\%, 0,6\%, 1,6\%, $0,2 \%$ y $0,15 \%$ (Andino y Pérez 2012)

La carencia de fósforo se manifiesta por retraso en la floración y baja producción de frutos y semillas. Y el exceso provoca fijación de zinc en el suelo, por tal razón la utilización de fósforo en el cultivo de maralfalfa es necesario para hacer producción animal a base de este pasto (Andino y Pérez 2012).

En los diferentes vegetales, principalmente en el maralfalfa, el potasio es el nutriente de mayor importancia en la producción de materia orgánica, interviene en el proceso de división celular regulando las disponibilidades de azúcares, de la misma manera en los procesos de absorción de $\mathrm{Ca}, \mathrm{N}$ y $\mathrm{Na}$, permite que la planta presente un buen vigor y resistencia a la presencia de enfermedades; una baja temperatura, ayuda a la producción de proteínas, se encarga del trasporte de azúcares desde las hojas al fruto (Andino y Pérez 2012), de esta manera la incorporación del fertilizante 10-3010 y gallinaza, hace que los suelos dispongan suficientes elementos macrominerales que permita una buena producción forrajera.

En relación con la producción de forraje verde por hectárea de Pennisetum $s p$, la utilización del tratamiento T3 permitió registrar $84.20 \mathrm{Tm} / \mathrm{ha}$, valor que difiere significativamente $(\mathrm{p}<0.01)$ de los tratamientos T0, T1 y T2 con los cuales se obtuvieron 42.10, 67.13 y $65.20 \mathrm{Tm} / \mathrm{ha}$ respectivamente. Al respecto, Cerda, R. (2014) al aplicar 90 $\mathrm{kg} /$ ha de nitrógeno, obtuvo una producción de 55,067 Tm de forraje verde, siendo inferior a los tratamientos alternativos aplicados en el presente trabajo. El mismo autor, señala que, una aplicación de $90 \mathrm{Kg} / \mathrm{ha}$ de nitrógeno, el pasto maralfalfa, registra un mayor valor de proteína cruda con $16,25 \%$.

Si bien es cierto, Cunuhay, J. y Choiloquinga, M. (2011) reporta que el cultivo del maralfalfa a una altura de $2900 \mathrm{msnm}$ con un suelo con un $\mathrm{pH}$ de 5.7, a los 150 días permite una producción de $74.4 \mathrm{Tm} / \mathrm{ha}$ de forraje verde y únicamente 2.46 cortes / año, 
siendo necesario determinar que la producción a nivel de sierra es más prolongada que a nivel de trópico húmedo, de esta manera se puede observar que es necesario estas investigaciones para tener calidad y cantidad de forraje en los diferentes pisos altitudinales.

La utilización del tratamiento (T3), permitió registrar una producción de 16.84 Tm de materia seca, valor que difiere significativamente del resto de tratamientos (T0, T1 y T2) con los cuales se reporta $8.42,13.43$ y $43.04 \mathrm{Tm} / \mathrm{ha}$ de materia seca. Mientras que Martínez et.al., (2010), citado por Condori, S. (2018) reportan producciones entre 7,2 y 9,2 Tm/ha de materia seca por corte a los 60 días cuando aplican fertilizantes foliares a base de boil, al igual que Cárdenas et.al., (2012) citado por Condori, S. (2018) a los 60 días obtuvieron producciones de 6,43 toneladas de materia seca. Condori, S. (2018) reporta que la producción de la Pennisetum sp en Choquenaira registró de 4,35 a 10,87 $\mathrm{Tm} /$ ha de materia seca en un ambiente controlado. Hinojoza, L. (2010) al determinar la producción de cuatro variedades de pasto de corte, entre ellas maralfalfa, este último registró una producción de 121,48 Tm de forraje verde/ha por año y una producción de 20,62 Tm de materia seca/ha/año.

Muñoz, A. (2004) citado por Erazo, C. (2009) manifiesta que, en las zonas con suelos pobres en materia orgánica, van de franco-arcillosos a franco-arenosos, en un clima relativamente seco, con $\mathrm{pH}$ de 4,5 a 5 a una altura aproximada de 1.750 m.s.n.m. y en lotes de tercer corte, reportan cosechas a los 75 días con una producción promedio de 28,5 kilos por metro cuadrado, es decir, 285 toneladas por hectárea, con una altura promedio por caña de $2,50 \mathrm{~m}$. Los cortes se deben realizar cuando el cultivo alcance aproximadamente un $10 \%$ de espigamiento (Arias, L. 2012), desde este punto de vista no esta tomando en consideración la digestibilidad principalmente de las paredes celulares (celulosa, hemicelusa y lignina) de los tallos.

Florián, (2015) citado por Velasteguí, M. (2018) menciona que el pasto maralfalfa, produce entre 200 y $400 \mathrm{Tm}$ por hectárea y esta determinada según el manejo de fertilizaciones y control de malezas, en lotes de tercer corte a 1750 m.s.n.m., se han obtenido cosechas a los 75 días con una producción de 285 toneladas por hectárea, con una altura promedio por caña de $2,50 \mathrm{~m}$, aunque también manifiesta que los cortes se realizan cuando la planta alcanza un metro de altura. Señala que el primer corte debe espigar, y los siguientes cuando el cultivo alcanza un 10\% de floración, posteriormente realizar cortes cada 40 días, de esta manera se observa que este pasto es precoz.

Cárdenas L, et al., (2012) al determinar la producción y calidad del pasto maralfalfa durante la época seca registra rendimientos de materia seca de 2,05 y 2,24 Tm/ha a los 30 y 60 días de crecimiento respectivamente, y de 15,76 Tm/ha a los 90 días de crecimiento luego de realizar la siembra en base a material vegetativo. Este último valor se asemeja al del presente estudio que en promedio para el caso de los tres tratamientos aplicados en promedio fue de $14,43 \mathrm{Tm}$. De materia seca por hectárea. 
cm a los 75 días; $173,50 \mathrm{~cm}$ a los 105 días y 212,67 cm a los 135 días,

Cruz, D. (2008) al realizar la evaluación del potencial forrajero del pasto maralfalfa con diferentes niveles de fertilización a base de Nitrógeno, Fósforo y Potasio, registró las mayores alturas de 133,17, 173,50 y 212,67 cm a los 75, 105 y 135 días respectivamente, también señala que la edad propicia para el corte de maralfalfa es recomendable a los 75 días, puesto a que, a esta edad existe una relación positiva entre la cantidad (184,93 Tm/FV/ha/año y calidad (16,70 \% MS y 15,30 \% PC) del forraje (Vargas, F. 2018), aunque falta análisis importantes como la digestibilidad de las paredes celulares, la materia seca y proteínas.

Rodríguez, R. (2014) reporta que el cultivo del paso maralfalfa se adapta alturas que van de 0 a $3000 \mathrm{msnm}$. Además, señala que es un cultivo forrajero perenne, el cual se siembra una sola vez y con el adecuado manejo después de terminar la temporada de lluvias y cortado el forraje, si el productor no dispone de agua para riego en la época de secas, llegada la temporada de lluvias vuelve a rebrotar y en caso de disponer de riego el productor puede obtener varios cortes por año. Es un cultivo que alcanza alturas de más de 4 metros. Su rendimiento en la región sur del Estado de México se obtiene únicamente en la temporada de lluvias variando su producción entre 150 a $200 \mathrm{Tm} / \mathrm{ha}$ de forraje verde y si dispone de riego en la temporada de estiaje la producción se duplica, siendo factibles de mejorar aun estos parámetros productivos con el manejo de cultivo. Es importante resaltar que un productor en pequeñas superficies puede obtener altos rendimientos, sin necesidad de sembrar grandes superficies, lo que redunda en que, el pasto mar alfalfa es una buena alternativa para los productores, que les permitirá mantener un buen número de animales en sus explotaciones y disminuir la compra de forrajes. Si queremos que la maralfalfa manifieste buenas producciones de forraje, calidad y sobre vivencia por varios años, es necesario atenderla como un cultivo que requiere cuidados y atenciones necesarias en cuanto a control de malezas, fertilización y manejo, aunque no tolera encharcamiento.

Tabla 1. Comportamiento productivo del pasto Maralfalfa por efecto de tres niveles de fertilizante.

\begin{tabular}{|c|c|c|c|c|c|c|c|c|c|c|}
\hline \multirow[b]{2}{*}{ Variables } & \multicolumn{8}{|c|}{ Tratamientos } & \multirow[b]{2}{*}{ Prob } & \multirow[b]{2}{*}{ E.E. } \\
\hline & TO & & $\mathrm{T} 1$ & & $\mathrm{~T} 2$ & & $\mathrm{~T} 3$ & & & \\
\hline Altura a los 30 días (m) & 0,54 & $\mathrm{~b}$ & 0,64 & $\mathrm{a}$ & 0,62 & & 0,66 & $\mathrm{a}$ & 0,00127 & 0,01 \\
\hline Altura a los 60 días (m) & 1,07 & $\mathrm{~b}$ & 1,28 & $\mathrm{a}$ & 1,24 & a & 1,31 & $\mathrm{a}$ & 0,00127 & 0,03 \\
\hline Altura a los 90 días (m) & 1,71 & $\mathrm{~b}$ & 2,03 & $\mathrm{a}$ & 1,96 & 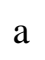 & 2,07 & $\mathrm{a}$ & 0,00127 & 0,04 \\
\hline Producción FV /m² (kg) & 4,21 & $\mathrm{c}$ & 6,71 & $\mathrm{~b}$ & 6,52 & U & 8,42 & $\mathrm{a}$ & 0,00010 & 0,34 \\
\hline Producción FV /Ha (Tm) & 42,10 & $\mathrm{c}$ & 67,13 & $\mathrm{~b}$ & 65,20 & b & 84,20 & $\mathrm{a}$ & 0,00010 & 3,42 \\
\hline Producción MS /Ha (Tm) & 8,42 & $\mathrm{c}$ & 13,43 & $\mathrm{~b}$ & 13,04 & $\mathrm{~b}$ & 16,84 & $\mathrm{a}$ & 0,00010 & 0,68 \\
\hline
\end{tabular}

Letras iguales horizontalmente, no difieren significativamente $(p>0.05)$. 


\section{Conclusiones}

- No se registraron diferencias estadísticas significativas entre los tratamientos alternativos respecto a la altura del pasto maralfalfa, por lo cual se recomienda la aplicación de fertilizante únicamente a base 10-30-10 + urea en dosis de 1000 y $2000 \mathrm{~kg} / \mathrm{ha} /$ corte respectivamente.

- La cantidad de forraje por metro cuadrado resulta ser excelente, razón por la cual se recomienda la utilización para pequeños, medianos y grandes ganaderos, no solamente en clima tropical sino también en el subtrópico como en ciertas zonas de la región interandina del Ecuador.

- Realizar análisis bromatólogo del pasto maralfafa cultivado con diferentes fertilizantes a diferentes edades y poder sugerir la edad optima de cosecha con una buena cantidad de nutrientes digeribles totales que apoyen al bienestar y nutrición animal.

\section{Referencias bibliográficas.}

Andino N.J., Pérez J.S. 2012. Producción de biomasa y concentración de nutrientes en el pasto cubano (Pennisetum purpureum x P. tiphoides) cv CT-115. Finca la Tigra, Cárdenas, Rivas, Nicaragua. Tesis para el título de Ing. en Zootecnia. Facultad de Ciencia Animal, Universidad Nacional Agraria, Nicaragua. 62p.

Arias, J. 2012. Comportamiento Agronómico Y Valor Nutricional De Tres Variedades De Pastos Pennisetum Para Corte En La Zona De Pichilingue Provincia De Los ríos "Autor, 74.

Calzada-Marín, J. M., Enríquez-Quiroz, J. F., Hernández Garay, A., Ortega Jiménez, E., \& Mendoza-Pedroza, S. I. 2014. Análisis de crecimiento del pasto maralfalfa (Pennisetum sp.) en clima cálido subhúmedo. Revista Mexicana de Ciencias Pecuarias, 5(2), 247. https://doi.org/10.22319/rmcp.v5i2.3664

Cárdenas L, Ruiz R, Medina F, Guevara f, Gomez H, Hernandez A, C. J. 2012. Producción y calidad del pasto maralfalfa (Pennisetum sp) durante la época seca. Universidad Autónoma de Chiapas. Facultad de Ciencias Agronómicas, 1(13), 3846. Retrieved from http://www.dgip.unach.mx/images/pdf-REVISTAQUEHACERCIENTIFICO/QUEHACER-CIENTIFICO-2012-enerjun/produccion_y_calidad_del_pasto_maralfalfa.pdf

Cerda, R. 2015. Comportamiento productivo del pasto maralfalfa (pennisetum sp.) con varias dosis de fertilización nitrogenada. Universidad de Costa Rica. Revista de las Sedes Regionales, vol. XVI, núm. 33, 2015, pp. 123-145.

Condori, S. C., Huanca, P. R., Guanto, O. T., \& Mendoza, G. J. C. 2018. Efficiency of water use and bromatological characteristics of maralfalfa (Pennisetum sp) under the application of bovine biol in the choquenaira experimental station, 5.

Corrales, M. Rda, F. Jaimez, R. Efecto del nitrógeno en los parámetros fotosintéticos y de producción del cultivo de la gerbera (Gerbera jamesonii H. Bolus ex Hook. f.). 
disponible en línea, <doi: http://dx.doi.org/10.15446/acag.v65n3.49555>. consulta (30/10/2020).

Correa H.J. 2006. Calidad nutricional del pasto maralfalfa (Pennisetum sp) cosechado a dos edades de rebrote. Facultad de Ciencias Agropecuarias, Universidad Nacional de Colombia, Sede Medellín, 13p.

Correa, H. Arroyave, Y. López, A. Ceron, J. 2015. Pasto Maralfalfa, Mitos y realidades. Dpto. de Producción Animal, Universidad Nacional de Colombia; Universidad Nacional de Colombia y COLANTA. https://www.engormix.com/ganaderiacarne/articulos/pasto-maralfalfa-t26119.htm

Cruz, D. 2008. Evaluación del potencial forrajero del pasto Maralfalfa Pennisetum violaceum con diferentes niveles de fertilización de nitrógeno y fósforo con a base estándar de potasio. Tesis de Grado. FCP - ESPOCH. Riobamba - Ecuador.

Cunuhay, J y Choloquinga, M. 2011. Evaluación de la adaptación del pasto Maralfalfa en dos pisos altitudinales con tres distancias de siembra en el campus Juan Lunardy y y Naste del cantón paute. Tesis, previa a la obtención de título Ingeniero Agropecuario. Universidad Politécnica Saleciana. Cuenca - Ecuador.

Duran, F. 2016. Pastos y Forrajes para Ganado. Edit. Grupo Latino. Impreso en Colombia. Erazo Villacrés, C. N. 2009. "Utilización De Ensilaje De Maralfalfa De Diferentes Edades De Corte (30,45 Y 60 Días) En La Alimentación De Cuyes,” 88. Retrieved from http://dspace.espoch.edu.ec/bitstream/123456789/2393/1/17T0894.pdf

Estrada, M. 2005. Manejo y Procesamiento de la Gallinaza. Facultad de Ciencias Administrativas y Agropecuarias de la Corporación Universitaria Lasallista. 6 p.

Fertiza, 2020. Información Técnica De Los Fertilizantes. Agroquímicos Fertiza. En linea. https://www.fertisa.com/pdf/productos/producto_11.pdf.

Hinojosa, Y. L. A., Yépez, N. D., Rodal, C. F., Ríos, O. A., Claros, B. R., Suárez, N. T., \& Jiménez, L. E. 2014. Producción y características agronómicas de cuatro variedades de pasto de corte del género Pennisetum, en Trinidad, Bolivia. Agrociencias Amazonia, 28-35.

Pacifex, 2018. Ficha Técnica. Fertilizantes Gavilon. http://innovacionagricola.com/wpcontent/uploads/2016/05/Urea-Pacifex-ficha-tecnica.pdf

Rincón A., Lagarreto G.A. 2010 Relación entre nitrógeno foliar y el contenido de clorofila, en maíz asociado con pastos en el Piedemonte Llanero colombiano. Revista Corpoica- Ciencia y Tecnología Agropecuaria, 11(2):122-128.

Rodríguez DR. 2014. Pasto Maralfalfa. Establecimiento, manejo y aprovechamiento en ganado caprino. México, DF. $15 \quad P$. recuperado de http://icamex.edomex.gob.mx/sites/icamex.edomex.gob.mx/files/files/publicaciones / 2014/pasto\%20maralfalfa.pdf.

Vargas, F. 2018. Valoración agro nutricional del pasto maralfalfa (pennisetum sp.) a dos tipos de fertilizantes en cuatro épocas de corte.

Velasteguí. M. 2018. Aplicación de fitohormonas como estimulantes de crecimiento del pasto maralfalfa (pennisetum sp.). tesis de grado. universidad de guayaquil facultad de ciencias agrarias carrera de ingeniería agronómica. En línea (consulta: 8 - 08 2020) 
<http://repositorio.ug.edu.ec/bitstream/redug/35568/1/Velastegu\%C3\%AD\%20Pun \%C3\%ADn\%20Miguel\%20\%C3\%81ngel.pdf>.

Yara, 2018. Nutrición vegetal. Solución vegetal. La gama de fertilizantes. https://www.yara.com.co/nutricion-vegetal/productos/ 


\section{PARA CITAR EL ARTÍCULO INDEXADO.}

Condo Plaza, L., Huebla Concha, V., Arias Alemán, L., \& Tapia Cabrera, N. (2021). Comportamiento productivo del pennisetum sp a la aplicación de 10-30-10, gallinaza y urea en el canton morona Santiago. ConcienciaDigital, 4(1.2), 256-268. https://doi.org/10.33262/concienciadigital.v4i1.2.1592

\section{¿Ciencia}

El artículo que se publica es de exclusiva responsabilidad de los autores y no necesariamente reflejan el pensamiento de la Revista Conciencia Digital.

El artículo queda en propiedad de la revista y, por tanto, su publicación parcial y/o total en otro medio tiene que ser autorizado por el director de la Revista Conciencia Digital.

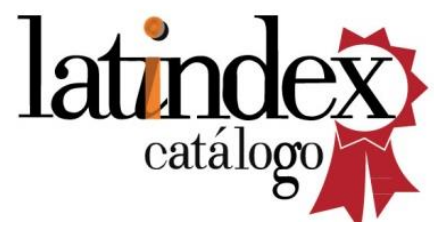

